Editor's Note: These short, critical reviews of recent papers in the Journal, written exclusively by graduate students or postdoctoral fellows, are intended to summarize the important findings of the paper and provide additional insight and commentary. For more information on the format and purpose of the Journal Club, please see http://www.jneurosci.org/misc/ifa_features.shtml.

\title{
Toward Understanding the Neurobiology of Social Attachment: Role of Estrogen Receptors in the Medial Amygdala
}

\author{
Viviana Trezza ${ }^{1}$ and Patrizia Campolongo ${ }^{2,3}$ \\ ${ }^{1}$ Rudolf Magnus Institute of Neuroscience, Department of Pharmacology and Anatomy, University Medical Center Utrecht, 3584CG Utrecht, The \\ Netherlands, ${ }^{2}$ Department of Biomedical Sciences, University of Foggia, 71100 Foggia, and ${ }^{3}$ Department of Physiology and Pharmacology, University of \\ Rome, 00159 Rome, Italy \\ Review of Cushing et al. (http://www.jneurosci.org/cgi/content/full/28/41/10399)
}

Social attachment is a complex construct that involves multisensory perception of social signals, higher cognitive functions such as attention and social recognition, and motivational processes. Understanding the neurobiological mechanisms and brain substrates of social attachment not only may be important in understanding normal emotions, such as love and separation, but also could aid our understanding and treatment of abnormal social behaviors that characterize several psychiatric diseases, such as autism, depression, and schizophrenia.

Prairie voles (Microtus ochrogaster) are an ideal animal model to study the neural substrates of social attachment. Prairie voles are small brown rodents that are found in grasslands in the central United States. They are monogamous: both males and females form enduring pair bonds and, after the death of one of the pair, $>70 \%$ of the surviving partners do not accept a new mate. Furthermore, unlike most other rodents, male prairie voles

Received 0ct. 28, 2008; revised Dec. 4, 2008; accepted Dec. 5, 2008.

Correspondence should be addressed to Dr. Patrizia Campolongo, Department of Physiology and Pharmacology, University of Rome, Piazzale Aldo Moro 5, 00159 Rome, Italy. E-mail: patrizia.campolongo@ uniroma1.it.

D0I:10.1523/JNEUROSC1.5193-08.2009

Copyright $\odot$ 2009 Society for Neuroscience $\quad 0270-6474 / 09 / 290001-02 \$ 15.00 / 0$ contribute significantly to nest building and show parental behaviors such as licking, grooming, huddling, and retrieving pups that wander from the nest.

Most studies in laboratory animals have pointed to two neuropeptides, oxytocin and vasopressin, as primary modulators of pair-bond formation and parental behaviors. In particular, it has been hypothesized that these neuropeptides link social information to reward circuits in the brain, leading to social attachment (Young et al., 2005). Several lines of evidence suggest an important role of estrogen receptor $\alpha(\mathrm{ER} \alpha)$ in this circuitry (Cushing and Kramer, 2005). First, studies using $\operatorname{ER} \alpha$ knock-out mice have shown that $\mathrm{ER} \alpha$ masculinizes behavior by reducing prosocial activities and inducing aggression. Second, male prairie voles, which are highly social, express low levels of $\mathrm{ER} \alpha$ in brain regions involved in social attachment, such as the medial amygdala (MeA), compared with male Kansas voles, which are significantly less social. Third, neonatal castration in prairie voles increases the expression of ER $\alpha$ in the MeA, which results in reduced male parental care and reduced ability of neuropeptides to stimulate pair bond formation. These studies indirectly suggest that changes in $\mathrm{ER} \alpha$ expression in specific brain areas highly affect social behavior and support a link between neuropeptides and estrogen in the neurobiology of social attachment. The MeA appears to play a crucial role in this process. To directly test this hypothesis, a selective increase in $\mathrm{ER} \alpha$ expression in the MeA must be shown to disrupt prosocial behaviors, such as male parental care and partner preference in prairie voles. This was the aim of a recent work published by Cushing et al. (2008) in The Journal of Neuroscience.

The authors' experimental design consisted of three groups of adult male prairie voles. A first group of animals was injected bilaterally in the MeA with adenoassociated viral vectors encoding human $\operatorname{ER} \alpha$, to achieve site-specific overexpression of $\mathrm{ER} \alpha$ in the MeA (MeA-ER $\alpha$ group). A second group of animals was injected in the same brain region with a similar vector encoding firefly luciferase cDNA (MeA site-specific control group), and a third group ( $\mathrm{ER} \alpha$ control group) was transfected with ER $\alpha$ in the caudateputamen, a brain region which does not express ER $\alpha$.

Male parental care and partner preference in prairie voles are among the best described examples of social attachment in rodents. Thus, 3 weeks after transfection, all prairie voles were tested by using 
the alloparental and social preference tests to evaluate whether the overexpression of $\mathrm{ER} \alpha$ in the MeA would result in changes in social attachment.

When given the opportunity to choose between an empty cage and a pup's cage, all MeA luciferase controls and caudate $\mathrm{ER} \alpha$ controls displayed parental behavior, defined as at least 3 min spent licking, grooming, or huddling (sitting with back arched) over the pup. However, compared with all control males, the majority of MeA-ER $\alpha$ males did not display parental behavior [Cushing et al. (2008), their Fig. 2 (http://www.jneurosci.org/cgi/ content/full/28/41/10399/F2)]. Instead, some $\mathrm{ER} \alpha$ males showed pup-directed aggression and, in two cases, pup-directed sexual activity.

Five days after the parental test, prairie voles were tested with the social preference test. The standard social preference test is based on the ability of pair-bonded animals to recognize and choose their mate over unfamiliar conspecifics and even to aggressively reject unfamiliar conspecifics from their territory. The testing apparatus consists of a central chamber connected with two identical chambers, one containing the partner and the other an unfamiliar conspecific. After mating or extensive cohabitation, prairie voles tested in this paradigm spend significantly longer time with their mate versus the unfamiliar conspecific. This behavior is referred to as a partner preference. In the protocol used by Cushing et al., experimental males were cohabited with sexually naive stimulus females only for $1 \mathrm{~h}$ before testing. Because females require longer cohabitation intervals to become sexually receptive, this protocol does not depend on previous mating and fails to produce partner preference. Indeed, as expected, MeA luciferase controls and caudate $\mathrm{ER} \alpha$ controls did not show social preference and spent equal time in contact with either the familiar or the novel female. In contrast, MeA-ER $\alpha$ males displayed unusual behavior, because they spent more time in the novel female cage than in the familiar female cage and more time in side-by-side contact with the novel female [Cushing et al. (2008), their Fig. 3 (http://www.jneurosci.org/ cgi/content/full/28/41/10399/F3)].

With their results, Cushing et al. show that increasing $\mathrm{ER} \alpha$ in the MeA not only disrupts the two main expressions of social attachment in prairie voles but also induces misdirected social behaviors, such as pup-directed sexual activity and preference for a novel female. Although prairie voles have been extensively used to study social attachment, this is the first study reporting such a peculiar change in their social behavior. Thus far, most studies on the neurobiology of social attachment have shown that neuropeptidergic mechanisms are primarily responsible for social-bond formation. Thus, central administration of neuropeptides stimulates paternal behavior and social-bond formation in prairie voles, whereas neuropeptide antagonists inhibit paternal activities and prevent mating-induced partner preference (Wang et al., 1994; Cho et al., 1999). However, pup-directed aggression and/or preference for a novel female has never been reported in male prairie voles after treatment with neuropeptide antagonists. Cushing et al. do not negate the well documented role of neuropeptides in social attachment but show that changing the pattern of expression of $\operatorname{ER} \alpha$ in the $\mathrm{MeA}$ alters the first steps involved in social-bond formation.

By integrating olfactory inputs from the olfactory bulbs into neuropeptidergic projections to the ventral pallidum and lateral septum, the MeA is a crucial link between the social behavior neural circuit and the reward system, whose simultaneous activation leads to social attachment (Young et al., 2005). The present results extend this understanding and show that the MeA might play a major role in the interaction between neuropeptides and estrogen through activation of $\operatorname{ER} \alpha$. The bed nucleus of the stria terminalis (BST) might also be involved in this process. The BST has reciprocal connections to the MeA and the medial preoptic area and sends neuropeptidergic fibers to the lateral septum. It has been suggested that, like that in the MeA, ER $\alpha$ expression in the BST is inversely correlated with male prosocial behavior (Cushing and Kramer, 2005). However, direct evidence that $\mathrm{ER} \alpha$ expression in the BST plays a role in social-bond formation is still missing and needs further investigation.

Other facets of social attachment could be modulated by $\operatorname{ER} \alpha$ activation. In a recent paper, Bosch et al. (2008) showed that male prairie voles separated from their female partners display passive stress-coping behavior in the forced swimming and tail-suspension tests. These effects were blocked by intracerebroventricular infusion of a corticotrophin-releasing factor (CRF) antagonist. This result, together with the observation that pair-bonded males show upregulation of CRF mRNA in the BST, suggests that activation of CRF system af- ter separation from the partner may be important to preserve the established pair. Estrogen, neuropeptide, and CRF receptors colocalize in several brain areas mediating social behavior. Thus, it is possible that an interaction between these neurochemical systems modulates the stress responses to the loss of a bonded partner in male prairie voles. Indeed, a complex process like social attachment is under the control of many neurochemical systems. Current research shows that the pattern of expression of estrogen, neuropeptide, and CRF receptors in specific brain areas modulates critical aspects of social behavior. Rather than functioning independently, these systems likely interact and share some common mechanisms in the regulation of pair bonding.

The results by Cushing et al. offer important new insights into the neuroanatomy and neurochemistry of social attachment by providing the first direct evidence that $\mathrm{ER} \alpha$ expression in the MeA plays a critical role in the expression of male prairie vole social behavior. Whereas these results confirm the importance of prairie voles as ideal animal models to study social attachment, the future challenge of social neuroscience research will be to translate these findings to human social bonding, especially in relation to neuropsychiatric disorders characterized by an inability to form normal social bonds, such as autism.

\section{References}

Bosch OJ, Nair HP, Ahern TH, Neumann ID, Young LJ (2008) The CRF system mediates increased passive stress-coping behavior following the loss of a bonded partner in a monogamous rodent. Neuropsychopharmacology. Retrieved December 12, 2008. doi:10.1038/npp.2008.154

Cho MM, DeVries AC, Williams JR, Carter CS (1999) The effects of oxytocin and vasopressin on partner preferences in male and female prairie voles (Microtus ochrogaster). Behav Neurosci 113:1071-1079.

Cushing BS, Kramer KM (2005) Mechanisms underlying epigenetic effects of early social experience: the role of neuropeptides and steroids. Neurosci Biobehav Rev 29:1089-1105.

Cushing BS, Perry A, Musatov S, Ogawa S, Papademetriou E (2008) Estrogen receptors in the medial amygdala inhibit the expression of male prosocial behavior. J Neurosci 28:10399-10403.

Wang Z, Ferris CF, De Vries GJ (1994) Role of septal vasopressin innervation in paternal behavior in prairie voles (Microtus ochrogaster). Proc Natl Acad Sci U S A 91:400-404.

Young LJ, Murphy Young AZ, Hammock EA (2005) Anatomy and neurochemistry of the pair bond. J Comp Neurol 493:51-57. 